PROCEEDINGS OF THE

AMERICAN MATHEMATICAL SOCIETY

Volume 137, Number 6, June 2009, Pages 1933-1939

S 0002-9939(08)09730-X

Article electronically published on December 29, 2008

\title{
NEW RESULTS ON THE LEAST COMMON MULTIPLE OF CONSECUTIVE INTEGERS
}

\author{
BAKIR FARHI AND DANIEL KANE
}

(Communicated by Ken Ono)

\begin{abstract}
When studying the least common multiple of some finite sequences of integers, the first author introduced the interesting arithmetic functions $g_{k}$ $(k \in \mathbb{N})$, defined by $g_{k}(n):=\frac{n(n+1) \ldots(n+k)}{\operatorname{lcm}(n, n+1, \ldots, n+k)}(\forall n \in \mathbb{N} \backslash\{0\})$. He proved that for each $k \in \mathbb{N}, g_{k}$ is periodic and $k$ ! is a period of $g_{k}$. He raised the open problem of determining the smallest positive period $P_{k}$ of $g_{k}$. Very recently, S. Hong and Y. Yang improved the period $k$ ! of $g_{k}$ to $\operatorname{lcm}(1,2, \ldots, k)$. In addition, they conjectured that $P_{k}$ is always a multiple of the positive integer $\frac{\operatorname{lcm}(1,2, \ldots, k, k+1)}{k+1}$. An immediate consequence of this conjecture is that if $(k+1)$ is prime, then the exact period of $g_{k}$ is precisely equal to $\operatorname{lcm}(1,2, \ldots, k)$.

In this paper, we first prove the conjecture of S. Hong and Y. Yang and then we give the exact value of $P_{k}(k \in \mathbb{N})$. We deduce, as a corollary, that $P_{k}$ is equal to the part of $\operatorname{lcm}(1,2, \ldots, k)$ not divisible by some prime.
\end{abstract}

\section{INTRODUCTION}

Throughout this paper, we let $\mathbb{N}^{*}$ denote the set $\mathbb{N} \backslash\{0\}$ of positive integers.

Many results concerning the least common multiple of sequences of integers are known. The most famous is none other than an equivalent of the prime number theorem; it states that $\log \operatorname{lcm}(1,2, \ldots, n) \sim n$ as $n$ tends to infinity (see, e.g., 6]). Effective bounds for $\operatorname{lcm}(1,2, \ldots, n)$ were also given by several authors (see, e.g., [5] and [10]).

Recently, the topic has undergone important developments. In 11, Bateman, Kalb and Stenger obtained a quantity equivalent to $\log \operatorname{lcm}\left(u_{1}, u_{2}, \ldots, u_{n}\right)$ for when $\left(u_{n}\right)_{n}$ is an arithmetic progression. In [2, Cilleruelo obtained a simple analog of the least common multiple for a quadratic progression. For the effective bounds, Farhi [3, 4] found lower bounds for $\operatorname{lcm}\left(u_{0}, u_{1}, \ldots, u_{n}\right)$ in the cases where $\left(u_{n}\right)_{n}$ is an arithmetic progression and where it is a quadratic progression. In the case of arithmetic progressions, Hong and Feng [7] and Hong and Yang [8] obtained some improvements of Farhi's lower bounds.

Among arithmetic progressions, the sequences of consecutive integers are the most well-known with regard to properties of their least common multiple. In 4,

Received by the editors July 28, 2008, and, in revised form, August 17, 2008.

2000 Mathematics Subject Classification. Primary 11A05.

Key words and phrases. Least common multiple, arithmetic function, exact period.

(C)2008 American Mathematical Society Reverts to public domain 28 years from publication 
Farhi introduced the arithmetic function $g_{k}: \mathbb{N}^{*} \rightarrow \mathbb{N}^{*}(k \in \mathbb{N})$, which is defined by

$$
g_{k}(n):=\frac{n(n+1) \ldots(n+k)}{\operatorname{lcm}(n, n+1, \ldots, n+k)}\left(\forall n \in \mathbb{N}^{*}\right) .
$$

Farhi proved that the sequence $\left(g_{k}\right)_{k \in \mathbb{N}}$ satisfies the recursive relation

$$
g_{k}(n)=\operatorname{gcd}\left(k !,(n+k) g_{k-1}(n)\right) \quad\left(\forall k, n \in \mathbb{N}^{*}\right) .
$$

Then, using this relation, he deduced (by induction on $k$ ) that for each $k \in \mathbb{N}, g_{k}$ is periodic and $k$ ! is a period of $g_{k}$. A natural open problem, raised in [4, is to determine the exact period (i.e., the smallest positive period) of $g_{k}$.

In the following, let $P_{k}$ denote the exact period of $g_{k}$. So, Farhi's result amounts to saying that $P_{k}$ divides $k$ ! for all $k \in \mathbb{N}$. Very recently, Hong and Yang have shown that $P_{k}$ divides $\operatorname{lcm}(1,2, \ldots, k)$. This improves Farhi's result but doesn't solve the problem of determining the $P_{k}$ 's. In their paper [8], Hong and Yang also conjectured that $P_{k}$ is a multiple of $\frac{\operatorname{lcm}(1,2, \ldots, k+1)}{k+1}$ for all nonnegative integers $k$. According to the property that $P_{k}$ divides $\operatorname{lcm}(1,2, \ldots, k)(\forall k \in \mathbb{N})$, this conjecture implies that the equality $P_{k}=\operatorname{lcm}(1,2, \ldots, k)$ holds at least when $(k+1)$ is prime.

In this paper, we first prove the conjecture of Hong and Yang and then give the exact value of $P_{k}(\forall k \in \mathbb{N})$. As a corollary, we show that $P_{k}$ is equal to the part of $\operatorname{lcm}(1,2, \ldots, k)$ which is not divisible by some prime and that the equality $P_{k}=\operatorname{lcm}(1,2, \ldots, k)$ holds for an infinite number of $k \in \mathbb{N}$ for which $(k+1)$ is not prime.

\section{Proof of the conjecture of Hong and Yang}

We begin by extending the functions $g_{k}(k \in \mathbb{N})$ to $\mathbb{Z}$ as follows:

- We define $g_{0}: \mathbb{Z} \rightarrow \mathbb{N}^{*}$ by $g_{0}(n)=1, \forall n \in \mathbb{Z}$.

- If, for some $k \geq 1, g_{k-1}$ is defined, then we define $g_{k}$ by the relation

$$
g_{k}(n)=\operatorname{gcd}\left(k !,(n+k) g_{k-1}(n)\right)(\forall n \in \mathbb{Z}) .
$$

These extensions are easily seen to be periodic and to have the same period as their restrictions to $\mathbb{N}^{*}$. The following proposition plays a vital role in what follows.

Proposition 2.1. For any $k \in \mathbb{N}$, we have $g_{k}(0)=k !$.

Proof. This follows by induction on $k$ upon using the relation (11).

We now arrive at the theorem implying the conjecture of Hong and Yang.

Theorem 2.2. For all $k \in \mathbb{N}$, we have

$$
P_{k}=\frac{\operatorname{lcm}(1,2, \ldots, k+1)}{k+1} \cdot \operatorname{gcd}\left(P_{k}+k+1, \operatorname{lcm}\left(P_{k}+1, P_{k}+2, \ldots, P_{k}+k\right)\right) .
$$

The proof of this theorem needs the following lemma:

Lemma 2.3. For all $k \in \mathbb{N}$, we have

$$
\operatorname{lcm}\left(P_{k}, P_{k}+1, \ldots, P_{k}+k\right)=\operatorname{lcm}\left(P_{k}+1, P_{k}+2, \ldots, P_{k}+k\right) .
$$

Proof of the lemma. Let $k \in \mathbb{N}$ be fixed. The required equality in the lemma is clearly equivalent to saying that $P_{k}$ divides $\operatorname{lcm}\left(P_{k}+1, P_{k}+2, \ldots, P_{k}+k\right)$. This amounts to showing that for any prime number $p$,

$$
v_{p}\left(P_{k}\right) \leq v_{p}\left(\operatorname{lcm}\left(P_{k}+1, \ldots, P_{k}+k\right)\right)=\max _{1 \leq i \leq k} v_{p}\left(P_{k}+i\right) .
$$


So it remains to show (2). Let $p$ be a prime number. As $P_{k}$ divides $\operatorname{lcm}(1,2, \ldots, k)$ (by the result of Hong and Yang [8]), we have $v_{p}\left(P_{k}\right) \leq v_{p}(\operatorname{lcm}(1,2, \ldots, k)$ ), that is, $v_{p}\left(P_{k}\right) \leq \max _{1 \leq i \leq k} v_{p}(i)$. So there exists $i_{0} \in\{1,2, \ldots, k\}$ such that $v_{p}\left(P_{k}\right) \leq$ $v_{p}\left(i_{0}\right)$. It follows, according to the elementary properties of the $p$-adic valuation, that we have

$$
v_{p}\left(P_{k}\right)=\min \left(v_{p}\left(P_{k}\right), v_{p}\left(i_{0}\right)\right) \leq v_{p}\left(P_{k}+i_{0}\right) \leq \max _{1 \leq i \leq k} v_{p}\left(P_{k}+i\right),
$$

which confirms (2) and completes this proof.

Proof of Theorem 2.2, Let $k \in \mathbb{N}$ be fixed. The main idea of the proof is to calculate in two different ways the quotient $\frac{g_{k}\left(P_{k}\right)}{g_{k}\left(P_{k}+1\right)}$ and then to compare the results obtained. On the one hand, we have, from the definition of the function $g_{k}$,

$$
\begin{aligned}
\frac{g_{k}\left(P_{k}\right)}{g_{k}\left(P_{k}+1\right)} & =\frac{P_{k}\left(P_{k}+1\right) \ldots\left(P_{k}+k\right)}{\operatorname{lcm}\left(P_{k}, P_{k}+1, \ldots, P_{k}+k\right)} / \frac{\left(P_{k}+1\right)\left(P_{k}+2\right) \ldots\left(P_{k}+k+1\right)}{\operatorname{lcm}\left(P_{k}+1, P_{k}+2, \ldots, P_{k}+k+1\right)} \\
& =P_{k} \frac{\operatorname{lcm}\left(P_{k}+1, P_{k}+2, \ldots, P_{k}+k+1\right)}{\left(P_{k}+k+1\right) \operatorname{lcm}\left(P_{k}, P_{k}+1, \ldots, P_{k}+k\right)} .
\end{aligned}
$$

Next, using Lemma 2.3 and the well-known formula " $a b=\operatorname{lcm}(a, b) \operatorname{gcd}(a, b)$ $\left(\forall a, b \in \mathbb{N}^{*}\right)$ ", we have

$$
\begin{aligned}
\left(P_{k}+k+1\right) \operatorname{lcm}\left(P_{k}\right. & \left., P_{k}+1, \ldots, P_{k}+k\right)=\left(P_{k}+k+1\right) \operatorname{lcm}\left(P_{k}+1, P_{k}+2, \ldots, P_{k}+k\right) \\
& =\operatorname{lcm}\left(P_{k}+k+1, \operatorname{lcm}\left(P_{k}+1, \ldots, P_{k}+k\right)\right) \\
& \times \operatorname{gcd}\left(P_{k}+k+1, \operatorname{lcm}\left(P_{k}+1, \ldots, P_{k}+k\right)\right) \\
=\operatorname{lcm}\left(P_{k}+1, P_{k}\right. & \left.+2, \ldots, P_{k}+k+1\right) \operatorname{gcd}\left(P_{k}+k+1, \operatorname{lcm}\left(P_{k}+1, \ldots, P_{k}+k\right)\right) .
\end{aligned}
$$

By substituting this into (3), we obtain

$$
\frac{g_{k}\left(P_{k}\right)}{g_{k}\left(P_{k}+1\right)}=\frac{P_{k}}{\operatorname{gcd}\left(P_{k}+k+1, \operatorname{lcm}\left(P_{k}+1, \ldots, P_{k}+k\right)\right)} \text {. }
$$

On the other hand, according to Proposition 2.1 and the definition of $P_{k}$, we have

$$
\frac{g_{k}\left(P_{k}\right)}{g_{k}\left(P_{k}+1\right)}=\frac{k !}{g_{k}(1)}=\frac{\operatorname{lcm}(1,2, \ldots, k+1)}{k+1} .
$$

Finally, by comparing (4) and (5), we get

$$
P_{k}=\frac{\operatorname{lcm}(1,2, \ldots, k+1)}{k+1} \operatorname{gcd}\left(P_{k}+k+1, \operatorname{lcm}\left(P_{k}+1, P_{k}+2, \ldots, P_{k}+k\right)\right),
$$

as required. The proof is complete.

From Theorem 2.2, we derive the following interesting corollary, which confirms the conjecture of Hong and Yang 8 .

Corollary 2.4. For all $k \in \mathbb{N}$, the exact period $P_{k}$ of $g_{k}$ is a multiple of the positive integer $\frac{\operatorname{lcm}(1,2, \ldots, k, k+1)}{k+1}$. In addition, for all $k \in \mathbb{N}$ such that $(k+1)$ is prime, we have precisely $P_{k}=\operatorname{lcm}(1,2, \ldots, k)$.

Proof. The first part of the corollary immediately follows from Theorem 2.2, Furthermore, we remark that if $k$ is a natural number such that $(k+1)$ is prime, then we have $\frac{\operatorname{lcm}(1,2, \ldots, k+1)}{k+1}=\operatorname{lcm}(1,2, \ldots, k)$. So, $P_{k}$ is both a multiple and a divisor of $\operatorname{lcm}(1,2, \ldots, k)$. Hence $P_{k}=\operatorname{lcm}(1,2, \ldots, k)$. This finishes the proof of the corollary. 
Now, we exploit the identity in Theorem 2.2 in order to obtain the $p$-adic valuation of $P_{k}(k \in \mathbb{N})$ for most prime numbers $p$.

Theorem 2.5. Let $k \geq 2$ be an integer and let $p \in[1, k]$ be a prime number satisfying

$$
v_{p}(k+1)<\max _{1 \leq i \leq k} v_{p}(i) .
$$

Then we have

$$
v_{p}\left(P_{k}\right)=\max _{1 \leq i \leq k} v_{p}(i) .
$$

Proof. The identity in Theorem 2.2 implies the following equality:

(7) $v_{p}\left(P_{k}\right)=\max _{1 \leq i \leq k+1}\left(v_{p}(i)\right)-v_{p}(k+1)+\min \left\{v_{p}\left(P_{k}+k+1\right), \max _{1 \leq i \leq k}\left(v_{p}\left(P_{k}+i\right)\right)\right\}$.

Now, using hypothesis (6) of the theorem, we have

$$
\max _{1 \leq i \leq k+1}\left(v_{p}(i)\right)=\max _{1 \leq i \leq k}\left(v_{p}(i)\right)
$$

and

$$
\max _{1 \leq i \leq k+1}\left(v_{p}(i)\right)-v_{p}(k+1)>0 .
$$

According to (7), this last inequality implies that

$$
\min \left\{v_{p}\left(P_{k}+k+1\right), \max _{1 \leq i \leq k} v_{p}\left(P_{k}+i\right)\right\}<v_{p}\left(P_{k}\right) .
$$

Let $i_{0} \in\{1,2, \ldots, k\}$ be such that $\max _{1 \leq i \leq k} v_{p}(i)=v_{p}\left(i_{0}\right)$. Since $P_{k}$ divides $\operatorname{lcm}(1,2, \ldots, k)$, we have $v_{p}\left(P_{k}\right) \leq v_{p}\left(i_{0}\right)$, which in turn implies that $v_{p}\left(P_{k}+i_{0}\right) \geq$ $\min \left(v_{p}\left(P_{k}\right), v_{p}\left(i_{0}\right)\right)=v_{p}\left(P_{k}\right)$. Thus $\max _{1 \leq i \leq k} v_{p}\left(P_{k}+i\right) \geq v_{p}\left(P_{k}\right)$. It follows from (9) that

$$
\min \left\{v_{p}\left(P_{k}+k+1\right), \max _{1 \leq i \leq k} v_{p}\left(P_{k}+i\right)\right\}=v_{p}\left(P_{k}+k+1\right)<v_{p}\left(P_{k}\right) .
$$

So, we have

$$
\min \left(v_{p}\left(P_{k}\right), v_{p}(k+1)\right) \leq v_{p}\left(P_{k}+k+1\right)<v_{p}\left(P_{k}\right),
$$

which implies that

and then that

$$
v_{p}(k+1)<v_{p}\left(P_{k}\right)
$$

$$
v_{p}\left(P_{k}+k+1\right)=\min \left(v_{p}\left(P_{k}\right), v_{p}(k+1)\right)=v_{p}(k+1) .
$$

According to (10), it follows that

$$
\min \left\{v_{p}\left(P_{k}+k+1\right), \max _{1 \leq i \leq k} v_{p}\left(P_{k}+i\right)\right\}=v_{p}(k+1) .
$$

By substituting (8) and (11) into (7), we finally get

$$
v_{p}\left(P_{k}\right)=\max _{1 \leq i \leq k} v_{p}(i)
$$

as required. The theorem is proved.

Using Theorem 2.5, we can find infinitely many natural numbers $k$ such that $(k+1)$ is not prime and the equality $P_{k}=\operatorname{lcm}(1,2, \ldots, k)$ holds. The following corollary gives concrete examples of such numbers $k$. 
Corollary 2.6. If $k$ is an integer having the form $k=6^{r}-1(r \in \mathbb{N}, r \geq 2)$, then we have

$$
P_{k}=\operatorname{lcm}(1,2, \ldots, k)
$$

Consequently, there are infinitely many $k \in \mathbb{N}$ for which $(k+1)$ is not prime and the equality $P_{k}=\operatorname{lcm}(1,2, \ldots, k)$ holds.

Proof. Let $r \geq 2$ be an integer and let $k=6^{r}-1$. We have $v_{2}(k+1)=v_{2}\left(6^{r}\right)=r$, while $\max _{1 \leq i \leq k} v_{2}(i) \geq r+1\left(\right.$ since $\left.k \geq 2^{r+1}\right)$. Thus $v_{2}(k+1)<\max _{1 \leq i \leq k} v_{2}(i)$.

Similarly, we have $v_{3}(k+1)=v_{3}\left(6^{r}\right)=r$, while $\max _{1 \leq i \leq k} v_{3}(i) \geq r+1$ (since $\left.k \geq 3^{r+1}\right)$. Thus $v_{3}(k+1)<\max _{1 \leq i \leq k} v_{3}(i)$.

Finally, for any prime $p \in[5, k]$, we clearly have $v_{p}(k+1)=v_{p}\left(6^{r}\right)=0$ and $\max _{1 \leq i \leq k} v_{p}(i) \geq 1$. Hence $v_{p}(k+1)<\max _{1 \leq i \leq k} v_{p}(i)$.

This shows that the hypothesis of Theorem 2.5 is satisfied by any prime number $p$. Consequently, we have for any prime $p$ that $v_{p}\left(P_{k}\right)=\max _{1 \leq i \leq k} v_{p}(i)=$ $v_{p}(\operatorname{lcm}(1,2, \ldots, k))$. Hence $P_{k}=\operatorname{lcm}(1,2, \ldots, k)$, as required.

\section{Determination of the exact value of $P_{k}$}

Notice that Theorem 2.5 successfully computes the value of $v_{p}\left(P_{k}\right)$ for almost all primes $p$ (in fact we will prove in Proposition 3.3 that Theorem 2.5 fails to provide this value for at most one prime). In order to evaluate $P_{k}$, all we have left to do is to compute $v_{p}\left(P_{k}\right)$ for primes $p$ such that $v_{p}(k+1) \geq \max _{1 \leq i \leq k} v_{p}(i)$. In particular we will prove:

Lemma 3.1. Let $k \in \mathbb{N}$. If $v_{p}(k+1) \geq \max _{1 \leq i \leq k} v_{p}(i)$, then $v_{p}\left(P_{k}\right)=0$.

From this lemma the following result is immediate.

Theorem 3.2. We have for all $k \in \mathbb{N}$ :

$$
P_{k}=\prod_{p \text { prime, } p \leq k} p \begin{cases}0 & \text { if } v_{p}(k+1) \geq \max _{1 \leq i \leq k} v_{p}(i) \\ \max _{1 \leq i \leq k} v_{p}(i) & \text { otherwise. }\end{cases}
$$

In order to prove this result, we need to look into some of the more detailed divisibility properties of $g_{k}(n)$. In this spirit we make the following definitions.

Let $S_{n, k}=\{n, n+1, n+2, \ldots, n+k\}$ be the set of integers in the range $[n, n+k]$.

For a prime number $p$, let $g_{p, k}(n):=v_{p}\left(g_{k}(n)\right)$. Let $P_{p, k}$ be the exact period of $g_{p, k}$. Since a positive integer is uniquely determined by the number of times each prime divides it, $P_{k}=\operatorname{lcm}_{p}$ prime $\left(P_{p, k}\right)$.

Now note that

$$
\begin{aligned}
g_{p, k}(n) & =\sum_{m \in S_{n, k}} v_{p}(m)-\max _{m \in S_{n, k}} v_{p}(m) \\
& =\sum_{e>0, m \in S_{n, k}}\left(1 \text { if } p^{e} \mid m\right)-\sum_{e>0}\left(1 \text { if } p^{e} \text { divides some } m \in S_{n, k}\right) \\
& =\sum_{e>0} \max \left(0, \#\left\{m \in S_{n, k}: p^{e} \mid m\right\}-1\right) .
\end{aligned}
$$

Let $e_{p, k}=\left\lfloor\log _{p}(k)\right\rfloor=\max _{1 \leq i \leq k} v_{p}(i)$ be the largest exponent of a power of $p$ that is at most $k$. Clearly there is at most one element of $S_{n, k}$ which is divisible by $p^{e}$ if 
$e>e_{p, k}$; therefore terms in the above sum with $e>e_{p, k}$ are all 0. Furthermore, for each $e \leq e_{p, k}$, at least one element of $S_{p, k}$ is divisible by $p^{e}$. Hence we have that

$$
g_{p, k}(n)=\sum_{e=1}^{e_{p, k}}\left(\#\left\{m \in S_{n, k}: p^{e} \mid m\right\}-1\right) .
$$

Note that each term on the right-hand side of (12) is periodic in $n$ with period $p^{e_{p, k}}$ since the condition $p^{e} \mid(n+m)$ for fixed $m$ is periodic with period $p^{e}$. Therefore $P_{p, k} \mid p^{e_{p, k}}$. Note that this implies that the $P_{p, k}$ for different $p$ are relatively prime, and hence we have

$$
P_{k}=\prod_{p \text { prime, } p \leq k} P_{p, k}
$$

We are now ready to prove our main result.

Proof of Lemma 3.1. Suppose that $v_{p}(k+1) \geq e_{p, k}$. It clearly suffices to show that $v_{p}\left(P_{q, k}\right)=0$ for each prime $q$. For $q \neq p$ this follows immediately from the result that $P_{q, k} \mid q^{e_{q, k}}$. Now we consider the case $q=p$.

For each $e \in\left\{1, \ldots, e_{p, k}\right\}$, since $p^{e} \mid k+1$, it is clear that $\#\left\{m \in S_{n, k}: p^{e} \mid m\right\}=$ $\frac{k+1}{p^{e}}$, which implies (according to (12)) that $g_{k, n}$ is independent of $n$. Consequently, we have $P_{p, k}=1$ and hence $v_{p}\left(P_{p, k}\right)=0$, which completes our proof.

Note that a slightly more complicated argument allows one to use this technique to provide an alternate proof of Theorem 2.5.

We can also show that the result in Theorem 3.2 says that $P_{k}$ is basically $\operatorname{lcm}(1,2, \ldots, k)$.

Proposition 3.3. There is at most one prime $p$ such that $v_{p}(k+1) \geq e_{p, k}$. In particular, by Theorem 3.2 , $P_{k}$ is either $\operatorname{lcm}(1,2, \ldots, k)$ or $\frac{\operatorname{lcm}(1,2, \ldots, k)}{p^{e_{p}, k}}$ for some prime $p$.

Proof. Suppose that for two distinct primes $p, q \leq k$ we have $v_{p}(k+1) \geq e_{p, k}$ and $v_{q}(k+1) \geq e_{q, k}$. Then

$$
k+1 \geq p^{v_{p}(k+1)} q^{v_{q}(k+1)} \geq p^{e_{p, k}} q^{e_{q, k}}>\min \left(p^{e_{p, k}}, q^{e_{q, k}}\right)^{2}=\min \left(p^{2 e_{p, k}}, q^{2 e_{q, k}}\right) .
$$

But this would imply that either $k \geq p^{2 e_{p, k}}$ or $k \geq q^{2 e_{q, k}}$, thus violating the definition of either $e_{p, k}$ or $e_{q, k}$.

\section{REFERENCES}

[1] P. Bateman, J. Kalb, and A. Stenger, A limit involving least common multiples, Amer. Math. Monthly, 109 (2002), 393-394.

[2] J. Cilleruelo, The least common multiple of a quadratic sequence, preprint (2008).

[3] B. Farhi, Minorations non triviales du plus petit commun multiple de certaines suites finies d'entiers, C. R. Math. Acad. Sci. Paris, 341 (2005), 469-474. MR2180812 (2006g:11006)

[4] - Nontrivial lower bounds for the least common multiple of some finite sequences of integers, J. Number Theory, 125 (2007), 393-411. MR2332595 (2008i:11001)

[5] D. Hanson, On the product of the primes, Canad. Math. Bull., 15 (1972), 33-37. MR0313179 $(47: 1734)$

[6] G. H. Hardy and E. M. Wright, An Introduction to the Theory of Numbers, fifth ed., The Clarendon Press, Oxford Univ. Press, New York, 1979. MR568909 (81i:10002)

[7] S. Hong and W. Feng, Lower bounds for the least common multiple of finite arithmetic progressions, C. R. Math. Acad. Sci. Paris, 343 (2006), 695-698. MR 2284695(2007h:11004)

[8] S. Hong and Y. Yang, On the periodicity of an arithmetical function, C. R. Math. Acad. Sci. Paris, 346 (2008), 717-721. 
[9] _ Improvements of lower bounds for the least common multiple of finite arithmetic progressions, Proc. Amer. Math. Soc., 136 (2008), 4111-4114.

[10] M. Nair, On Chebyshev-type inequalities for primes, Amer. Math. Monthly, 89 (1982), 126129. MR643279 (83f:10043)

Département de Mathématiques, Université du Maine, Avenue Olivier Messiaen, 72085 Le Mans Cedex 9, France

E-mail address: bakir.farhi@gmail.com

Department of Mathematics, Harvard University, 1 Oxford Street, Cambridge, MasSACHUSETTS 02139

E-mail address: aladkeenin@gmail.com 